\section{Fibroma Traumático en Pacientes de Cirugía Bucal}

\author{
Traumatic Fibroma in Oral Surgery Patients
}

\section{Fibroma traumático em pacientes de cirurgia oral}

\author{
Iván Álvarez \\ ivan12alvarez@gmail.com \\ Luisana Morón \\ luisandrein22@outlook.com \\ Alberto Viloria \\ albertoviloriahern0815@gmail.com \\ Recibido enero 2019 / Revisión febrero 2019 / Aceptado 1 de mayo 2019
}

\section{RESUMEN}

Introducción: El fibroma traumático es un crecimiento tumoral benigno que aparece en cualquier órgano, con mayor frecuencia en la cavidad oral. Se define como, una hiperplasia reactiva de tejido conectivo fibroso, su factor etiológico es el traumatismo o la irritación local. Se presenta en la mucosa de revestimiento, bordes laterales de lengua y reborde alveolar de pacientes edéntulos. Objetivo: Determinar la prevalencia de Fibroma Traumático en pacientes del Área de Cirugía Bucal de la Universidad José Antonio Páez. Materiales y Métodos: Investigación descriptiva con un diseño de campo, observacional y retrospectivo, la población estuvo constituida por 1861 pacientes que acudieron al Área de Cirugía Bucal, se tomó una muestra intencional donde estuvieron incluidas las historias médicas de los pacientes a los que se le realizó una biopsia dentro de la cavidad bucal y con sospecha de fibroma traumático, sin distinción de edad, sexo y localización de la lesión, dicha muestra estuvo representada por 26 historias. Resultados: El sector con mayor prevalencia fue el de jóvenes y adultos, el sexo femenino, y el área anatómica el carrillo. Los aspectos morfológicos e histológicos de las lesiones coinciden con los reportados en la literatura. Conclusión: El adecuado diagnóstico clínico e histológico patológico, el manejo y el tratamiento son fundamentales, así como la educación al paciente sobre la importancia de una revisión odontológica.

Palabras clave: fibroma; traumático; cirugía bucal; odontología

\begin{abstract}
Introduction: Traumatic fibroid is a benign tumor growth that appears in any organ, most often in the oral cavity. It is defined as, a reactive hyperplasia of fibrous connective tissue, its etiological factor is trauma or local irritation. It occurs in the lining mucosa, lateral edges of the tongue and alveolar rim of edentulous patients. Objective: To determine the prevalence of Traumatic Fibroma in patients of the Oral Surgery Area of the José Antonio Páez University. Materials and Methods: Descriptive research with a field design, observational and retrospective, the population consisted of 1861 patients who attended the Oral Surgery Area, an intentional sample was taken which included the medical records of the patients who were performed a biopsy within the oral cavity and with suspicion of traumatic fibroma, without distinction of age, sex and location of the lesion, this sample was represented by 26 stories. Results: The sector with the highest prevalence was youth and adults, the female sex, and the cheek anatomical area. The morphological and histological aspects of the lesions coincide with those reported in the literature. Conclusion: Adequate clinical and pathological histological diagnosis, management and treatment are essential, as well as patient education on the importance of a dental review.
\end{abstract}

Key words: fibroma; traumatic; oral surgery; odontology
IA: Universidad Arturo Michelena, Venezuela.

LM: Universidad Arturo Michelena, Venezuela.

AV: Universidad Arturo Michelena, Venezuela. 


\section{RESUMO}

IA: Universidad Arturo Michelena, Venezuela.

LM: Universidad Arturo Michelena, Venezuela.

AV: Universidad Arturo Michelena, Venezuela.

\begin{abstract}
Introdução: O fibróide traumático é um crescimento benigno do tumor que aparece em qualquer órgão, mais frequentemente na cavidade oral. É definida como uma hiperplasia reativa do tecido conjuntivo fibroso, cujo fator etiológico é trauma ou irritação local. Ocorre na mucosa do revestimento, nas bordas laterais da língua e na borda alveolar de pacientes desdentados. Objetivo: Determinar a prevalência de Fibroma Traumático em pacientes da Área de Cirurgia Oral da Universidade José Antonio Páez. Materiais e Métodos: Pesquisa descritiva, com delineamento de campo, observacional e retrospectiva. A população foi composta por 1861 pacientes atendidos na Área de Cirurgia Oral, foi realizada uma amostra intencional que incluía os prontuários médicos dos pacientes atendidos. Realizada biópsia na cavidade oral e com suspeita de fibroma traumático, sem distinção de idade, sexo e localização da lesão, esta amostra foi representada por 26 histórias. Resultados: 0 setor com maior prevalência foi de jovens e adultos, o sexo feminino e a área anatômica da bochecha. Os aspectos morfológicos e histológicos das lesões coincidem com os relatados na literatura. Conclusão: $O$ diagnóstico, o manejo e o tratamento clínico e patológico histológico são essenciais, bem como a educação do paciente sobre a importância de uma revisão dentária.
\end{abstract}

Palavras-chave: fibróide; traumático; cirurgia oral; odontologia

\section{INTRODUCCIÓN}

\ a cavidad bucal, se encuentra constituida por sus paredes y las estructuras anexas que corresponden a los dientes, los labios superiores e inferiores, la lengua y las glándulas salivales. La boca propiamente dicha es el espacio que se extiende desde la cara lingual o posterior de los dientes, hasta el istmo de las fauces, atrás la boca presenta seis paredes: anterior, posterior, laterales, superior e inferior. La pared anterior está formada por la cara posterior o mucosa de los labios. La pared posterior está formada por el paladar blando (velo del paladar). Los carrillos conforman las paredes laterales, el paladar duro conforma la pared superior y el piso de la boca constituye la pared inferior (1).

La mucosa de revestimiento posee un epitelio plano estratificado no queratinizado, está en la cara interna de los labios, los carrillos, el piso de la boca, cara ventral de la lengua y el paladar blando. Estos sitios se encuentran tapizados por músculo estriado (labios, carrillos y lengua), hueso (mucosa alveolar) y glándulas (paladar blando, mejillas y superficie ventral de la lengua).
La mucosa de revestimiento tiene papilas menos abundantes que son más cortas para poder adaptarse a los movimientos de los músculos subyacentes (2).

La lengua como parte de la cavidad bucal, constituye un órgano muscular recubierto por una mucosa especializada que consiste en un epitelio plano estratificado queratinizado y una lámina propia densa, continuación de los tabiques conectivos que separan la musculatura estriada lingual. El aspecto rugoso de la mayor parte de su superficie dorsal de la región anterior se debe a la presencia de proyecciones denominadas papilas linguales, todas revestidas de epitelio escamoso estratificado y un núcleo central de tejido conectivo laxo (3).

En la cavidad bucal, se pueden encontrar diversas patologías o lesiones según sea su forma de aparición y origen como el producido por infecciones virales, malformaciones óseas, alteraciones cromosómicas, de procedencia traumática como un desorden en la oclusión que dará procedencia a los fibromas ya que son lesiones donde intervienen los malos hábitos al abrir y cerrar la boca, esta incorrecta práctica causa alteraciones a 
nivel macroscópico y microscópico. El fibroma es una de las patologías benignas más frecuente en la cavidad bucal, consta de tejido conectivo completamente desarrollado, presenta una localización superficial o profunda y los hay de distintos tipos, dependiendo de su origen pueden ser odontogénicos, no odontogénicos entre este último resalta el fibroma periférico o por irritación (4).

El fibroma traumático, también conocido como fibroma por irritación, fibroma oral, hiperplasia fibrosa local, hiperplasia fibrosa inflamatoria, cicatriz hiperplásica y pólipo fibroepitelial, es una lesión reactiva causada habitualmente por traumatismo crónico a las membranas de la mucosa bucal, es la lesión nodular más frecuente en la cavidad bucal, entre los estímulos asociados a esta patología figuran el hábito de mordisquearse los carrillos y labios, la actividad masticatoria incorrecta, la irritación por prótesis mal adaptadas (coronas, puentes fijos, prótesis parciales o removibles), las estructuras dentarias fracturadas y las restauraciones filosas (4).

Las áreas anatómicas más comunes para encontrar fibromas bucales son: área superior ò los lados de la lengua, el interior de las mejillas o en cualquier área de los labios. Además de la sensación y la apariencia, los fibromas orales no producen ningún síntoma. Se desarrollan durante semanas o meses hasta alcanzar un tamaño máximo, generalmente de aproximadamente $1 \mathrm{~cm}$ de diámetro, pero pueden ser más grandes. Suele ser una lesión solitaria, se deben considerar los diagnósticos asociados, que incluyen la esclerosis tuberosa, el síndrome de Cowden, la fibromatosis familiar y la hiperplasia papilar fibrótica del paladar $(5,6)$.

La patogénesis del fibroma oral está relacionada con su origen traumático. La lesión crónica inicialmente induce una inflamación, seguida por la reparación y si el agente causante persiste, se produce una sobre acumulación de colágeno producido por fibroblastos que origina la formación de tejido cicatricial A pesar que en un principio, se creía una neoplasia, hoy en día se define como una patología reactiva y no se considera un verdadero tumor, se caracteriza por la elaboración abundante de haces maduros de colágeno, razón por la cual en ocasiones se asemeja a tejido cicatricial (7).Histológicamente se caracteriza por mostrar un epitelio de revestimiento de tipo escamoso estratificado que se mantiene intacto, presenta hiperqueratosis por la fricción crónica de baja intensidad o por mostrar focos de ulceración cuando el trauma es intenso (8).

Según la OMS (9), entre el 16\% y el $40 \%$ de los niños entre 6 y 12 años padecen traumatismos bucodentales debidos a la falta de seguridad en los parques infantiles $\mathrm{y}$ escuelas, los accidentes de tránsito, actos de violencia, mala higiene, uso de: ortodoncia. En los adultos se ha reportado como causas de fibroma traumático los procedimientos de reparación de deficiente calidad, donde se han dejado estructuras dentarias punzantes $y / o$ cortantes al igual que aquellos pacientes que usen prótesis mal adaptadas que ocasionan trauma crónico del que deriva la aparición de fibroma (9).

La prevalencia de enfermedades bucodentales varía según la región geográfica, la disponibilidad y accesibilidad de servicios de salud bucodental. Para la OMS (9). Existe un aumento en los países de ingresos bajos y medianos, la morbilidad por esta causa es alta en las poblaciones pobres $\mathrm{y}$ desfavorecidas. Algunas investigaciones han reportado mayor prevalencia de lesiones del tejido conjuntivo y el diagnóstico histopatológico más frecuente es el del fibroma traumático (10). Este 
puede parecerse clínicamente a lesiones como el lipoma, mucocele, granuloma piogénico, fibroma de células gigantes, fibromas periféricos odontogénicos y hasta tumores de glándulas salivales, dependiendo de su ubicación. Por tal razón en imperativo realizar el estudio de la biopsia (11).

Macroscópicamente no es posible diferenciar y diagnosticar esta patología, es necesario que el gremio estomatológico desarrolle estudios a nivel histopatológicos, ya que los registros de enfermedades bucales de observación microscópica son escasos, es por ello que por todo lo planteado anteriormente, los investigadores consideran pertinente realizar la presente investigación con el objetivo de determinar la prevalencia del fibroma traumático a pacientes que asistieron al Área de Cirugía Bucal de la Universidad José Antonio Páez 2014-2017.

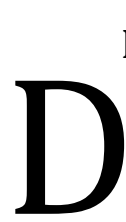

\section{MATERIALES Y MÉTODO}

iseño ex-post-facto, observacional debido a que el investigador se comporta como un espectador del fenómeno en estudio $(12,13)$. Es una investigación descriptiva donde se persigue la caracterización, registro, análisis e interpretación de la naturaleza actual $(14,15)$. El diseño es retrospectivo, debido a que se basa en asuntos, datos $\mathrm{u}$ observaciones ya pasados registrados en las historias clínicas de los pacientes (16).
La población 1861 pacientes que asistieron al Área de Cirugía Bucal de la Universidad José Antonio Páez comprendidos en el periodo 2014-2017. La muestra se obtuvo mediante muestreo intencional quedo constituida por 26 historias clínicas de pacientes que asistieron al Área de Cirugía Bucal, les fue removido el fibroma y se les practicó biopsia para diagnóstico confirmativo. Se seleccionaron las historias médicas de los pacientes a los que se les realizó una biopsia de la cavidad bucal y con posible fibroma traumático. El instrumento utilizado fue la ficha de registro que incluyó variables, definidas como una entidad abstracta que adquiere distintos valores, varía de un sujeto a otro, de manera que se considera como variable en esta investigación la prevalencia del fibroma traumático.

\section{RESULTADOS Y DISCUSIÓN}

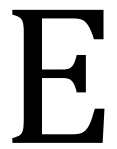

1 mayor número de pacientes que presento fibroma bucal traumático se ubicó en su mayoría $31 \%$ para las edades entre 26 a 41 años y $31 \%$ de 15 a 25 años, hubo un de $27 \%$ que se encontraba entre 42 y 57 años, quienes presentaron menor porcentaje fueron las personas entre 58 y 73 años de edad. De igual forma se puede presentar a cualquier edad, pero es más frecuente después de la segunda década de la vida, en individuos adultos. 


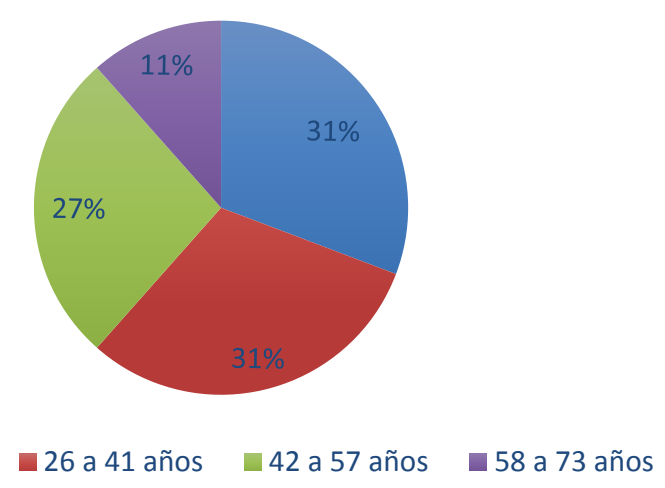

Gráfico 1. Distribución de pacientes con fibroma traumático bucal según edad. Área de Cirugía Bucal

Tabla 1. Distribución de pacientes según el sexo. Prevalencia de fibroma bucal traumático. Área de Cirugía Bucal.

\begin{tabular}{lll}
\hline Sexo & Fa & $\%$ \\
\hline Femenino & 17 & $65 \%$ \\
Masculino & 9 & $35 \%$ \\
Total & 26 & $100 \%$ \\
\hline
\end{tabular}

Fuente: instrumento aplicado

Del total de pacientes que presentaron fibroma traumático bucal, el 65\% correspondió al sexo femenino, mientras que $35 \%$ fue de sexo masculino. Hubo mayor prevalencia en el sexo femenino. Resultados que se corresponden con lo encontrado en la literatura donde se reporta una mayor prevalencia en mujeres que en hombres de esta patología

Tabla 2. Distribución de pacientes según antecedente odontológico. Prevalencia de fibroma bucal traumático. Área de Cirugía Bucal.

\begin{tabular}{llc}
\hline Antecedentes Odontológicos & Frecuencia & $\%$ \\
\hline Refiere & 11 & $42 \%$ \\
No Refiere & 15 & $58 \%$ \\
Total & 26 & $100 \%$ \\
\hline
\end{tabular}

Fuente: instrumento aplicado 
Se evidencia que el $42 \%$ de los pacientes previamente había recibido tratamientos odontológicos, esta información es de importancia para orientar el tratamiento, debido a que se puede inferir acerca de la relación que tienen estos antecedentes con la aparición de fibroma traumático, un trabajo que haya ocasionado algún tipo de molestia como la irritación por prótesis mal adaptadas (coronas, puentes fijos 0 dentaduras parciales removibles), aparatología ortodóntica, estructuras dentarias fracturadas y márgenes sobresalientes de las restauraciones, irritantes como: cálculo, placa, entre otros, pudieran ser el causante de la afección, tal como señala ampliamente la literatura científica.

Tabla 3. Distribución de pacientes según ubicación anatómica de la lesión. Prevalencia de fibroma bucal traumático. Área de Cirugía Bucal.

\begin{tabular}{lll}
\hline Ubicación Anatómica & Fa & \% \\
\hline Labio Superior & 3 & $12 \%$ \\
Labio Inferior & 4 & $15 \%$ \\
Lengua & 5 & $19 \%$ \\
Encías & 4 & $15 \%$ \\
Carrillos & 10 & $38 \%$ \\
Total & 26 & $100 \%$ \\
\hline
\end{tabular}

Fuente: instrumento aplicado

Los resultados destacan que los carrillos fueron las estructuras anatómicas más afectadas en 38\% de los pacientes, luego la lengua con $19 \%$ y labio inferior y encías con $15 \%$, el labio superior $12 \%$. La localización más frecuente del fibroma traumático, según la literatura y los resultados expresados en la tabla 3 es en aquellas áreas propensas a sufrir trauma, como, por ejemplo, carrillos, lengua, paladar y labio.

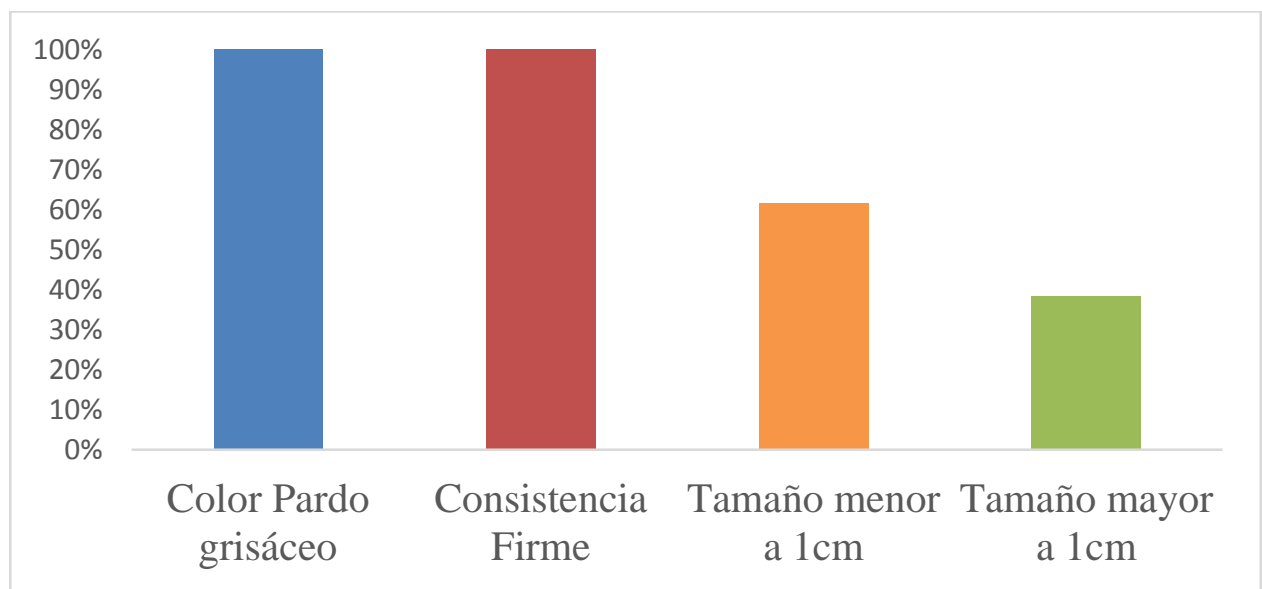

Gráfico 2. Distribución de pacientes según examen macroscópico. Prevalencia de fibroma bucal traumático. Área de Cirugía Bucal. 
El examen macroscópico orienta el diagnóstico, el grafico 2 da cuenta de las características encontradas en la población objeto de estudio en cuanto a color, consistencia y tamaño. El fibroma traumático bucal crece muy lentamente hasta alcanzar un tamaño determinado, en este grupo la mayoría se ubicó en menor de $1 \mathrm{~cm}$, la consistencia firme evidenciada depende de la cantidad y disposición de las fibras de colágeno.

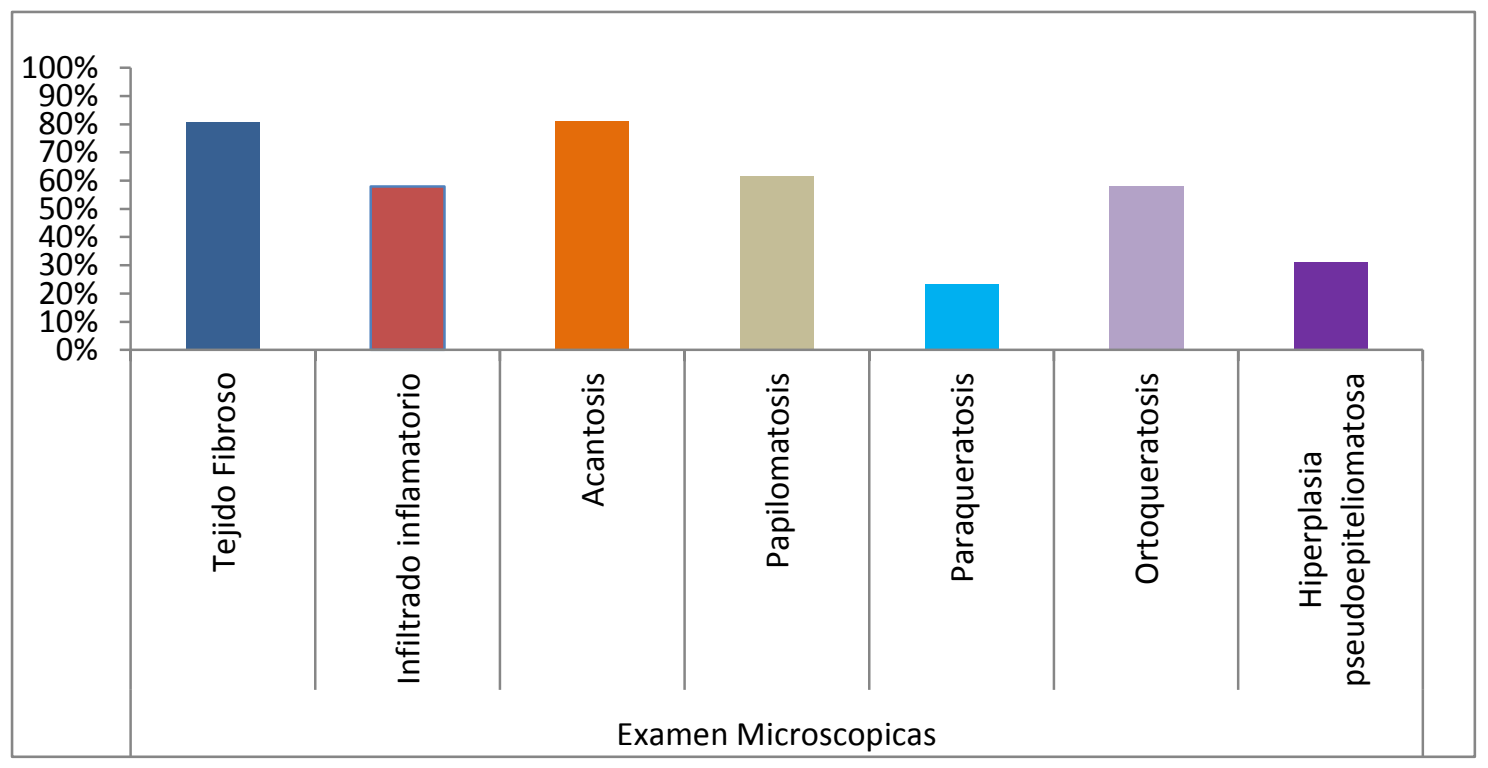

Gráfico 3. Distribución de pacientes según el examen microscópico. Prevalencia de fibroma bucal traumático. Área de Cirugía Bucal. Universidad José Antonio Páez 2014-2017

El resultado expresado en el grafico 3 evidencia la presencia de tejido fibroso, inflamatorio, acantosis y papilomatosis con mayor frecuencia en los pacientes estudiados, en menor proporción se encontró la paraqueratosis y la hiperplasia pseudoepiteliomatosa, datos característicos del fibroma traumático bucal, considerados de importancia debido a que el diagnóstico y tratamiento de la patología se basa en la correlación de hallazgos tanto clínicos como histopatológicos

En la presente investigación el sector más afectado por el fibroma bucal traumático según la edad del paciente se encontró en un rango que oscila entre los jóvenes y adultos se podría inferir que en este grupo de la población es donde ocurren sucesos rutinarios en el estilo de vida que puedan ocasionar lesiones, como, por ejemplo, los deportistas en la práctica de su disciplina. Este resultado contrasta con el obtenido en la investigación realizada por López (17) y Correa et al (18) que señalan que los picos de prevalencia se presentan en la cuarta y sexta décadas de la vida.

Algunos autores aseguran que tiene predominio por el sexo femenino $(4,17)$ tal como se encontró en la presente investigación donde un $65 \%$ de pacientes correspondía a este sexo, podría relacionarse con el hecho de que las mujeres son más cuidadosas de su imagen personal.

Los antecedentes odontológicos son un factor a tomar en cuenta para la aparición del fibroma bucal traumático. Un procedimiento deficiente puede ser el causante de la afección, entre ellos, ortodoncia, reparación de caries y extracciones son los principales en las 3 primeras décadas de vida, mientras que los 
usos de prótesis mal adaptadas en adultos mayores provocarían un estímulo crónico en zonas anatómicas como lo son la lengua, encía y los carrillos (4).

Refiere Somacarrera (19) que en algunos estudios los pacientes ancianos presentaron lesiones asociadas a prótesis en el $45 \%$ de los casos, y aproximadamente el $60 \%$ de estos pacientes presentaba alguna lesión mucosa no asociada al uso de prótesis. Los pacientes portadores de dentaduras completas suelen presentar más lesiones que los pacientes portadores de dentaduras parciales removibles

Para Rocafuerte (20) el fibroma bucal traumático se encuentra generalmente en la mucosa del carrillo, seguido de la mucosa labial, con menos frecuencia se puede encontrar en la cara dorsal de la lengua, se presenta como un crecimiento único y rara vez mide más de 1,5 centímetros. Estos planteamientos coinciden con los hallazgos en pacientes del Área de Cirugía Bucal de la Universidad José Antonio Páez donde se evidenció según los registros clínicos que el área anatómica afectada con más frecuencia eran los carrillos con un crecimiento que no pasaba de un centímetro.

Desde el punto de vista histológico el fibroma bucal traumático se presenta como una masa nodular sólida, no encapsulada, cubierta por epitelio escamoso estratificado, tejido conectivo denso con presencia de colágeno tipo I y III, dispuesto al azar y en ocasiones tejido conectivo fibroso hialinizado. Puede haber infiltrado inflamatorio crónico que contiene en su mayoría linfocitos y células plasmáticas (20). En la revisión efectuada en la presente investigación se encontró concordancia con el diagnóstico morfológico de la patología como lo es tejido fibroso, acantosis, papilomatosis, ortoqueratosis e infiltrado inflamatorio. Para López (17) la histopatología de esta lesión muestra una importante proliferación de fibras colágenas dispuestas al azar y entremezcladas con fibroblastos ampliamente dispersos en la matriz densa de colágeno, fibrocitos y pequeños vasos sanguíneos. Si la lesión se presenta traumatizada, en el tejido se puede observar vasodilatación, edema e infiltración de células inflamatorias crónicas.

\section{CONCLUSIÓN}

$\mathrm{E}$ l fibroma bucal traumático es una entidad con una alta prevalencia, entre mujeres y en la segunda y tercera etapa de la vida, que requiere de una correlación de hallazgos tanto clínicos como histopatológicos para tratar a estos pacientes. La eliminación de la fuente de irritación es importante para evitar la recurrencia de las lesiones y se requiere un seguimiento continuo posterior al tratamiento quirúrgico de la lesión. La prevención, el adecuado diagnóstico clínico e histológico patológico, el manejo $y$ el tratamiento son fundamentales. La educación al paciente sobre la importancia de una revisión odontológica habitual, al momento de presentar una lesión por un accidente traumático para estudiar detenidamente la zona afectada y la evolución de la lesión, evitar hábitos que lesionan la cavidad oral, podría disminuir la prevalencia de esta lesión.

El odontólogo tiene un papel de primer orden para el correcto diagnóstico y tratamiento del fibroma bucal traumático y también para evitar ocasionar lesiones cuando realiza procedimientos odontológicos

- Los autores declaran no poseer conflictos de interés.

- Financiamiento: autofinanciado. 
- Apegado a las normativas bioéticas para la publicación de trabajos de investigación.

- Agradecimientos: ninguno declarado por los autores.

\section{REFERENCIAS BIBLIOGRÁFICAS}

1. Pró E. Anatomía Clínica .1e. Buenos Aires: Medica Panamericana; 2012: 218-223

2. Ross M. Histología: Texto y Atlas a color con biología celular y molecular. $6^{\underline{a}}$ ed. Madrid: Médica Panamericana; 2013

3. Neils N. Netter Anatomía de Cabeza y Cuello para Odontólogos. 2ta ed. Barcelona: Elsevier Mason; 2012

4. Suárez V, Vanegas S. Fibroma traumático evaluación clínica e histológica de un caso. Acta Bioclinica (Internet). 2012 (citado el 16 nov. 2017) 2017; 1(1):1. Disponible en: http://erevistas.saber.ula.ve/index.php /actabioclinica/article/view/3360

5. Mesa M, Vázquez D, Rodríguez M. Presentación de un paciente con fibroma traumático subyacente a prótesis dental. CCM [Internet]. 2013 (citado 2017 junio); 17(4): 523527. Disponible en: http://scielo.sld.cu/scielo.php?script=sc i_arttext\&pid=S1560 -

43812013000400014\&lng=es

6. Pérez-Salcedo L, Bascones A. Tumores benignos de la mucosa oral. Av. Odontoestomatol (Internet). 2010 (citado 2017 junio); 26(1): 11-18. Disponible en: http://scielo.isciii.es/scielo.php?script= sci_arttext\&pid=S021312852010000100002\&lng=es

7. Regezi J, Sciubba J. Patología Bucal.3a e. Buenos Aires: McGraw-Hill Interamericana; 2007: 203-241

8. Marchena L, Luna M, Cruz D, Fernández C. Cirugía bucal de un fibroma traumático. Gaceta dental: Industria y profesiones, 2015(citado el 16 nov. 2017) ;(266):126-131. Disponible en https://gacetadental.com/2015/02/cir ugia-bucal-de-un-fibroma-traumatico52447/
9. Centro de Prensa de la OMS. Salud bucodental [Internet]. Organización Mundial de la Salud. 2012 (citado 3 noviembre 2017). Disponible en: https://www.who.int/es/newsroom/fact-sheets/detail/oral-health

10. Moret Y. Prevalencia de lesiones benignas y potencialmente malignas que afectan la mucosa bucal en pacientes adultos. Facultad de Odontología UCV. Acta Odontológica Venezolana. (Internet). 2014(citado el 16 nov. 2017); 52(1). [Internet] Disponible en: https://www.actaodontologica.com/edi ciones/2014/1/art-15/

11. Pinedo F, Fernández A, Bravo C, Somarriva C. Fibroma Odontogénico Periférico: Reporte de un Caso. Int. J. Odontostomat. (Internet). 2017 (citado 17 junio 2017); 11(2): 178-181.doi.org/10.4067/S0718381X20170002000

12. Hernández R, Fernández C. Metodología de la Investigación. México: McGraw Hill; 2014

13. Hurtado J. Metodología de la Investigación: Guía para la comprensión holística de la Ciencia.4 $4^{\mathrm{a}}$ ed. Caracas: Quirón Ediciones; 2010

14. Sousa D, Driessnack, Mendes I. Revisión de diseños de investigación resaltantes para enfermería. Parte 1: diseños de investigación cuantitativa. Rev. LatinoAm. Enfermagem (Internet). 2007 (citado junio 2017); 15(3): 502507.doi.org/10.1590/S010411692007000300022

15. Palella, S y Martins, F. Metodología De La Investigación Cuantitativa. Venezuela: FEDEUPEL; 2012

16. Pineda, E y Alvarado, E. Metodología de la Investigación. (3 $3^{\mathrm{a}}$ ed.). Washington: Organización Panamericana de Salud; 2008

17. López E. Estudio epidemiológico del fibroma traumático en una muestra de la población venezolana durante el período 1991-2001. Acta Odontológica Venezolana (Internet).2009; 47(3). Disponible en: https://www.actaodontologica.com/edi ciones/2009/3/art-11/ 
18. Correa P, Arias S. Resección de fibroma en mucosa oral. Técnica del estrangulamiento. Rev. CES Odont (Internet) 2016(citado 02 julio 2017); 29(1): 82-87. Disponible en: http://www.scielo.org.co/pdf/ceso/v 29n1/v29n1a09.pdf

19. Somacarrera M, López A., Martín C, Díaz M. Lesiones traumáticas en la mucosa oral de los adultos mayores.
Av. Odontoestomatol (Internet). 2015 (citado 02 julio 2017); 31(3): 129-134. doi.org/10.4321/S021312852015000300003

20. Rocafuerte A. Fibroma traumático en cavidad oral -una revisión. KIRU (Internet). 2017(citado 15 junio 2017); 16(1):41-46. doi.org/10.24265/kiru.2018.v16n1.07 